isms was associated with infection, and all 6 patients survived. Vancomycin-resistant enterococci or other vancomycin-resistant gram-positive organisms were not found in 663 patients treated with vancomycin for documented gram-positive infections or in 1,027 patients where perioperative vancomycin was used. It was concluded that the use of vancomycin as the primary therapeutic agent in seriously burned patients was not associated with increased risk of VRE isolation or VRE infection.

FROM: McManus AT, Goodwin CW, Pruitt BA Jr. Observations on the risk of resistance with the extended use of vancomycin. Arch Surg 1998;133:1207-1211.

\section{Survival of Enterococci on Dry Surfaces}

Wendt and coinvestigators from the Institute of Hygiene, Free University, Berlin, compared the abilities of Enterococcus faecium strains (three vancomycin-resistant enterococci [VRE] and five vancomycin-susceptible enterococci [VSE]) and Enterococcus faecalis strains (one VRE and $10 \mathrm{VSE}$ ) to survive under dry conditions. All strains survived for at least 1 week, and two strains survived for 4 months. Survival was not associated with the species ( $E$ faecalis vs $E$ faecium), the source of isolation (patient vs environment), or the susceptibility to vancomycin (VRE vs VSE). Resistance to dry conditions may promote the transmissibility of a strain, but VRE have no advantages over VSE with respect to their ability to survive under dry conditions.

FROM: Wendt C, Wiesenthal B, Dietz E, Rüden H. Survival of vancomycin-resistant and vancomycin-susceptible enterococci on dry surfaces. J Clin Microbiol 1998;36:3734-3736.

\section{Abbreviated Regimens of Zidovudine Reduce Risk of Perinatal HIV Transmission}

The Pediatric AIDS Clinical Trials Group Protocol 076 reported a reduction in the rate of perinatal transmission of HIV from $25.5 \%$ to $8.3 \%$ with a three-part regimen of zidovudine given antepartum, intrapartum, and to the newborn. Wade and colleagues from the New York State Department of Health and the University of Albany, New York, School of Public Health, recently examined the effects of abbreviated zidovudine regimens on perinatal HIV transmission using data from the HIV polymerase chain reaction (PCR) testing service of the New York State Department of Health.

Rates of perinatal HIV transmission varied, depending on when zidovudine prophylaxis was begun. When treatment was begun in the prenatal period, the rate of HIV transmission was $6.1 \%$ (95\% confidence interval, $4.1 \%$ -
$8.9 \%)$; when begun intrapartum, the rate was $10.0 \%(3.3 \%$ $21.8 \%$; when begun within the first 48 hours of life, the rate was $9.3 \%(4.1 \%-17.5 \%)$; and when begun on day 3 of life or later, the rate was $18.4 \%(7.7 \%-34.3 \%)$. In the absence of zidovudine prophylaxis, the rate of HIV transmission was $26.6 \%$ (21.1\%-32.7\%).

These results confirm the efficacy of zidovudine prophylaxis and suggest that there are reductions in the rates of perinatal transmission of HIV even with the use of abbreviated regimens that are begun intrapartum or in the first 48 hours of life. These abbreviated preventive regimens may be particularly suitable for use in parts of the world where the prevalence of HIV infection is high and resources are severely limited.

FROM: Wade NA, Birkhead GS, Warren BL, Charbonneau TT, French PT, Wang L, et al. Abbreviated regimens of zidovudine prophylaxis and perinatal transmission of the human immunodeficiency virus. $N$ Engl J Med 1998;339:1409-1414.

\section{Reducing Vancomycin Use Utilizing a Computer Guideline}

Minimizing vancomycin use represents a key strategy in preventing the spread of vancomycin-resistant enterococci. Shojania and colleagues from Brigham and Women's Hospital, Boston, conducted a study of a computerized guideline to reduce vancomycin use. At the time of physician order entry into the computer, the screen displayed an adaptation of the CDC's guidelines for appropriate vancomycin use. The main outcome measures were the frequency of initiation and renewal of vancomycin therapy and the duration of therapy.

The study included 396 physicians and 1,798 patients in a tertiary-care teaching hospital. Compared with the control group, intervention physicians wrote $32 \%$ fewer orders (11.3 vs 16.7 orders/physician; $P=.04$ ) and had $28 \%$ fewer patients for whom they either initiated or renewed an order for vancomycin ( 7.4 vs 10.3 orders/physician; $P=.02$ ). In addition, the duration of vancomycin therapy attributable to physicians in the intervention group was $36 \%$ lower than the duration of therapy prescribed by control physicians (26.5 vs 41.2 days; $P=05$ ). Pharmacy data confirmed a decrease in the overall hospital use of intravenous vancomycin during the study period. The authors concluded that implementation of a computerized guideline using physician order entry decreased vancomycin use. Computerized guidelines represent a promising tool for changing prescribing practices.

FROM: Shojania KG, Yokoe D, Platt R, Fiskio J, Ma'luf $\mathrm{N}$, Bates DW. Reducing vancomycin use utilizing a computer guideline: results of a randomized controlled trial. J Am Med Inform Assoc 1998;5(6):554-562. 